\title{
Intersecting Branes, Defect Conformal Field Theories and Tensionless Strings
}

\author{
Neil R. Constable ${ }^{a}$, Johanna Erdmenger ${ }^{b}$, Zachary Guralnik ${ }^{b}$ and Ingo Kirsch $b$ f \\ ${ }^{a}$ Center for Theoretical Physics and Laboratory for Nuclear Science \\ Massachusetts Institute of Technology \\ 77 Massachusetts Avenue \\ Cambridge, MA 02139, USA \\ ${ }^{b}$ Institut für Physik \\ Humboldt-Universität zu Berlin \\ Invalidenstraße 110 \\ D-10115 Berlin, Germany
}

\begin{abstract}
The defect conformal field theory describing intersecting D3-branes at a $\mathbb{C}^{2} / \mathbb{Z}_{k}$ orbifold is used to (de)construct the theory of intersecting M5-branes, as well as M5-branes wrapping the holomorphic curve $x y=c$. The possibility of a 't Hooft anomaly due to tensionless strings at the intersection is discussed. This note is based on a talk given by Zachary Guralnik at the 35th International Symposium Ahrenshoop on the Theory of Elementary Particles.
\end{abstract}

*constabl@lns.mit.edu, jke@physik.hu-berlin.de, zack@physik.hu-berlin.de, ik@physik.hu-berlin.de 


\section{Introduction}

String theory has led to the discovery of many novel interacting conformal theories. Amongst these are the "defect" conformal theories which describe intersecting branes at low energies. Such theories were studied originally in [1, 2, 3] and more recently in the context of AdS/CFT duality in [4, 5, 6, 7, 8, 9, 10, 11, 12, 13, 14.

One of the more exotic examples of this type of theory is the one which arises for intersecting M5-branes. Because of the difficulty of constructing a non-abelian generalization of a chiral two-form, a Lagrangian description is lacking even for parallel M5-branes. The dynamics of intersecting M5-branes is even richer and less understood, due to the presence of tensionless strings localized at the intersection [15]. Until very recently, the only concrete formulation of the theory was in terms of a discrete light cone quantization using the matrix model discussed in [16].

In this note, we shall describe an alternate formulation, in which the theory of the M5-M5 intersection is obtained from a limit of a defect conformal field theory with twodimensional $(4,0)$ supersymmetry [17]. This formulation is a natural extension of the "(de)construction" of the six-dimensional theory of parallel M5-branes discussed in 18 , 19. The $(4,0)$ defect CFT describes the low energy limit of intersecting D3-branes at a $\mathbb{C}^{2} / \mathbb{Z}_{k}$ orbifold. In a suitable $k \rightarrow \infty$ limit on the Higgs branch, the two extra dimensions of the M5-M5 intersection are generated.

We shall begin by discussing the $(4,4)$ defect CFT which describes D3-branes intersecting in flat space. This theory is the low energy limit of the M5-M5 intersection on a torus. We shall present the exact superpotential of the $(4,4)$ defect CFT in $(2,2)$ superspace. The resolution of the intersection to the holomorphic curve $x y=c$ can be seen explicitly from solutions of the F-flatness conditions. Upon orbifolding to a $(4,0)$ theory and taking the appropriate limit, all the degrees of freedom with momentum on the torus are generated.

In $(2,0)$ superspace, the analogue of a superpotential for the $(4,0)$ defect CFT is readily obtained from the superpotential for the $(4,4)$ defect CFT. At the appropriate point on the Higgs branch, this superpotential can be interpreted as lattice kinetic term in a compact direction, generating a Kaluza-Klein spectrum in the $k \rightarrow \infty$ limit. Via the S-duality of the model, a Kaluza-Klein spectrum on a two-torus is generated. In the continuum limit, the two-dimensional fields localized at the intersection correspond to tensionless strings propagating in the four common dimensions of the M5-M5 intersection.

The $(4,0)$ defect $\mathrm{CFT}$ has a $S U(2)_{L} \mathrm{R}$-symmetry. In the continuum limit, we shall argue that this becomes the $S U(2)$ R-symmetry of the M5-M5 intersection, which preserves four-dimensional $\mathcal{N}=2$ supersymmetry. The $S U(2)_{L}$ R-symmetry exhibits a 't Hooft anomaly. In the continuum limit, this may indicate an anomaly in the $S U(2)$ R-symmetry due to tensionless strings. While local $S U(2)$ anomalies do not arise in local four-dimensional quantum field theories, the possibility is not excluded for a fourdimensional theory of tensionless strings. 


\section{The D3-D3 intersection in flat space}

Consider a stack of $N$ parallel D3-branes in the directions 0123 intersecting an orthogonal stack of $N^{\prime}$ D3'-branes in the directions 0145. The action has the form $S=S_{\mathrm{D} 3}+S_{\mathrm{D} 3^{\prime}}+$ $S_{\mathrm{D} 3-\mathrm{D} 3^{\prime}}$. The components $S_{\mathrm{D} 3}$ and $S_{\mathrm{D} 3}$ each correspond to a four-dimensional $\mathcal{N}=4$ theory. The term $S_{\mathrm{D} 3-\mathrm{D} 3}$ contains couplings to a two-dimensional $(4,4)$ hypermultiplet, leaving only $(4,4)$ supersymmetry unbroken. The action was explicitly constructed in $(2,2)$ superspace in [14], to which we refer the reader for a more detailed discussion.

It is convenient to define the coordinates $z^{ \pm}=X^{0} \pm X^{1}, x=X^{2}+i X^{3}$ and $y=X^{4}+$ $i X^{5}$. The two-dimensional $(2,2)$ superspace is spanned by $\left(z^{+}, z^{-}, \theta^{+}, \theta^{-}, \bar{\theta}^{+}, \bar{\theta}^{-}\right)$. The four-dimensional fields corresponding to D3-D3 strings are described by $(2,2)$ superfields with extra continuous labels $x, \bar{x}$, while fields associated to the D3'-D3' strings have the extra labels $y, \bar{y}$. Although the four-dimensional parts of the action will look unusual in $(2,2)$ superspace, this notation makes sense since only a two-dimensional supersymmetry is preserved. The fields associated with D3-D3' strings are trapped at the intersection and have no extra continuous label.

Let us first consider $S_{\mathrm{D} 3}$, which involves $(2,2)$ superfields of the form $F\left(z^{+}, z^{-}, \theta, \bar{\theta} \mid x, \bar{x}\right)$. The $(2,2)$ fields appearing in this action are a vector superfield $V$, together with three adjoint chiral superfields $Q_{1}, Q_{2}$ and $\Phi$. The gauge connections $A_{0,1}$ of the $(2,2)$ vector multiplet and the complex scalar $\phi$ of the $(2,2)$ chiral field $\Phi$ combine to give the four components of the four-dimensional gauge connection. The scalar components of $V, Q_{1}$ and $Q_{2}$ combine to give the six adjoint scalars of the four-dimensional $\mathcal{N}=4$ theory. The field content of the second D3-brane (D3') is identical to that of the first D3-brane with the replacements $x \rightarrow y, V \rightarrow \mathcal{V}, Q_{i} \rightarrow S_{i}$ and $\Phi \rightarrow \Upsilon$. The fields corresponding to D3-D3' strings are the chiral multiplets $B$ and $\tilde{B}$ in the $\left(N, \bar{N}^{\prime}\right)$ and $\left(\bar{N}, N^{\prime}\right)$ representations of the $S U(N) \times S U\left(N^{\prime}\right)$ gauge group. Together $B$ and $\tilde{B}$ form a $(4,4)$ hypermultiplet.

For simplicity, we just present the superpotential, $W=W_{D 3}+W_{D 3^{\prime}}+W_{D 3-D 3^{\prime}}$, where

$$
\begin{aligned}
& W_{\mathrm{D} 3}=\int d^{2} x \epsilon_{i j} \operatorname{tr} Q_{i}\left[\partial_{\bar{x}}+g \Phi, Q_{j}\right], \quad W_{\mathrm{D} 3^{\prime}}=\int d^{2} y \epsilon_{i j} \operatorname{tr} S_{i}\left[\partial_{\bar{y}}+g \Upsilon, S_{j}\right] \\
& W_{\mathrm{D} 3-\mathrm{D} 3^{\prime}}=\frac{i g}{2} \operatorname{tr}\left(B \tilde{B} Q_{1}-\tilde{B} B S_{1}\right)
\end{aligned}
$$

The Lorentz invariance of the four-dimensional parts of the action $S_{D 3}$ or $S_{D 3}$ is not manifest in $(2,2)$ superspace, but can seen upon integrating out auxiliary fields. Consider the Lorentz invariant kinetic term for the scalar component $q_{1}$ of the chiral superfield $Q_{1}$, $\operatorname{tr} \partial_{\mu} \bar{q}_{1} \partial^{\mu} q_{1}$ with $\mu=0,1,2,3$. This term arises from a combination of a $(2,2)$ Kähler term $\operatorname{tr} \bar{Q}_{1} Q_{1}$ and the superpotential term $\operatorname{tr} Q^{1} \partial_{z} Q^{2}$.

On the classical Higgs branch the scalar components $b$ and $\tilde{b}$ of the chiral fields $B$ and $\tilde{B}$ have non-zero expectation values. The scalar components $s_{2}$ and $q_{2}$ of the chiral superfields $S_{2}$ and $Q_{2}$ also have expectation values given by the vanishing of the F-terms

\footnotetext{
${ }^{1}$ The procedure of writing supersymmetric $d$-dimensional theories in terms of a lower dimensional superspace has been discussed in various places [20, 8, 21, 14].
} 
for $S^{1}$ and $Q^{1}$ :

$$
\frac{\partial W}{\partial q_{1}}=\partial_{\bar{x}} q_{2}-g \delta^{2}(x) b \tilde{b}=0, \quad \frac{\partial W}{\partial s_{1}}=\partial_{\bar{y}} s_{2}-g \delta^{2}(y) \tilde{b} b=0
$$

With the geometric identifications $q_{2} \sim y / \alpha^{\prime}$ and $s_{2} \sim x / \alpha^{\prime}$, the solutions of these equations give rise to holomorphic curves of the form $x y=c \alpha^{\prime}$, when $2 \pi i c=g b \tilde{b}=g \tilde{b} b$.

\section{Intersecting D3-branes at a $\mathbb{C}^{2} / \mathbb{Z}_{k}$ orbifold}

The $(4,4)$ defect CFT which describes intersecting D3-branes in flat space has an $S U(2)_{L} \times$ $S U(2)_{R}$ R-symmetry. Geometrically, this corresponds to a rotation of the four coordinates transverse to all the D3-branes. These four directions can be described by two complex coordinates $u$ and $w$. We shall now consider the intersecting D3-branes in the orbifold geometry defined by $\left(u, w^{*}\right) \sim \exp (2 \pi i / k)\left(u, w^{*}\right)$.

The action in this background can be obtained from that of the $(4,4)$ defect CFT with $U(N k) \times U\left(N^{\prime} k\right)$ gauge group by projecting out degrees of freedom which are not invariant under the $\mathbb{Z}_{k}$ orbifold action. The $\mathbb{Z}_{k}$ orbifold is embedded in a combination of the gauge symmetry and the $S U(2)_{R}$ R-symmetry. The result of the projection is a $(4,0)$ defect CFT, with $U(N)^{k} \times U\left(N^{\prime}\right)^{k}$ gauge group and an $S U(2)_{L}$ R-symmetry. The field content and gauge transformation properties are summarized by the quiver diagram in figure 1. The inner and outer rings of the quiver diagram separately correspond to the $\mathcal{N}=2, d=4$ superconformal Yang Mills theory which was used in 18 to (de)construct the six-dimensional $(2,0)$ theory describing parallel M5-branes. The "spokes" stretching between the inner and outer rings correspond to the degrees of freedom localized at the two dimensional intersection. These degrees of freedom descend from the $(2,2)$ chiral superfields $B$ and $\tilde{B}$ of the parent $(4,4)$ theory, and consist of $(2,0)$ chiral multiplets $B_{j}$ and $\tilde{B}_{j}$ as well as $(2,0)$ Fermi multiplets $\Lambda_{j, j+1}^{B}$ and $\Lambda_{j, j+1}^{\tilde{B}}$. The indices $j$ label nodes of the quiver diagram and run from 1 to $k$. In an appropriate limit, the "spoke" fields will be seen to correspond to the tensionless strings of the M5-M5 intersection.

\section{Generating two extra dimensions}

In the $(4,0)$ defect CFT, two extra dimensions are generated at a point on the Higgs branch where the bifundamental scalars of the inner and outer rings of the quiver diagram have equal non-zero expecation values. This can be seen directly from the analogue of a $(2,0)$ superpotential for the $(4,0)$ defect CFT. We shall focus on the part of the superpotential involving the degrees of freedom at the intersection, which is given by

$$
\begin{aligned}
W_{\mathrm{D} 3-\mathrm{D} 3^{\prime}} & =g \operatorname{tr}_{N \times N}\left(\Lambda_{j, j+1}^{B}\left(\tilde{B}_{j+1} Q_{j, j+1}^{1}-S_{j+1, j}^{1} \tilde{B}_{j}\right)+\Lambda_{j}^{Q^{1}} B_{j} \tilde{B}_{j}\right) \\
& +\left.g \operatorname{tr}_{N^{\prime} \times N^{\prime}}\left(\Lambda_{j, j+1}^{\tilde{B}}\left(Q_{j+1, j}^{1} B_{j}-B_{j+1} S_{j+1, j}^{1}\right)-\Lambda_{j}^{S^{1}} \tilde{B}_{j} B_{j}\right)\right|_{\bar{\theta}^{+}=0}
\end{aligned}
$$



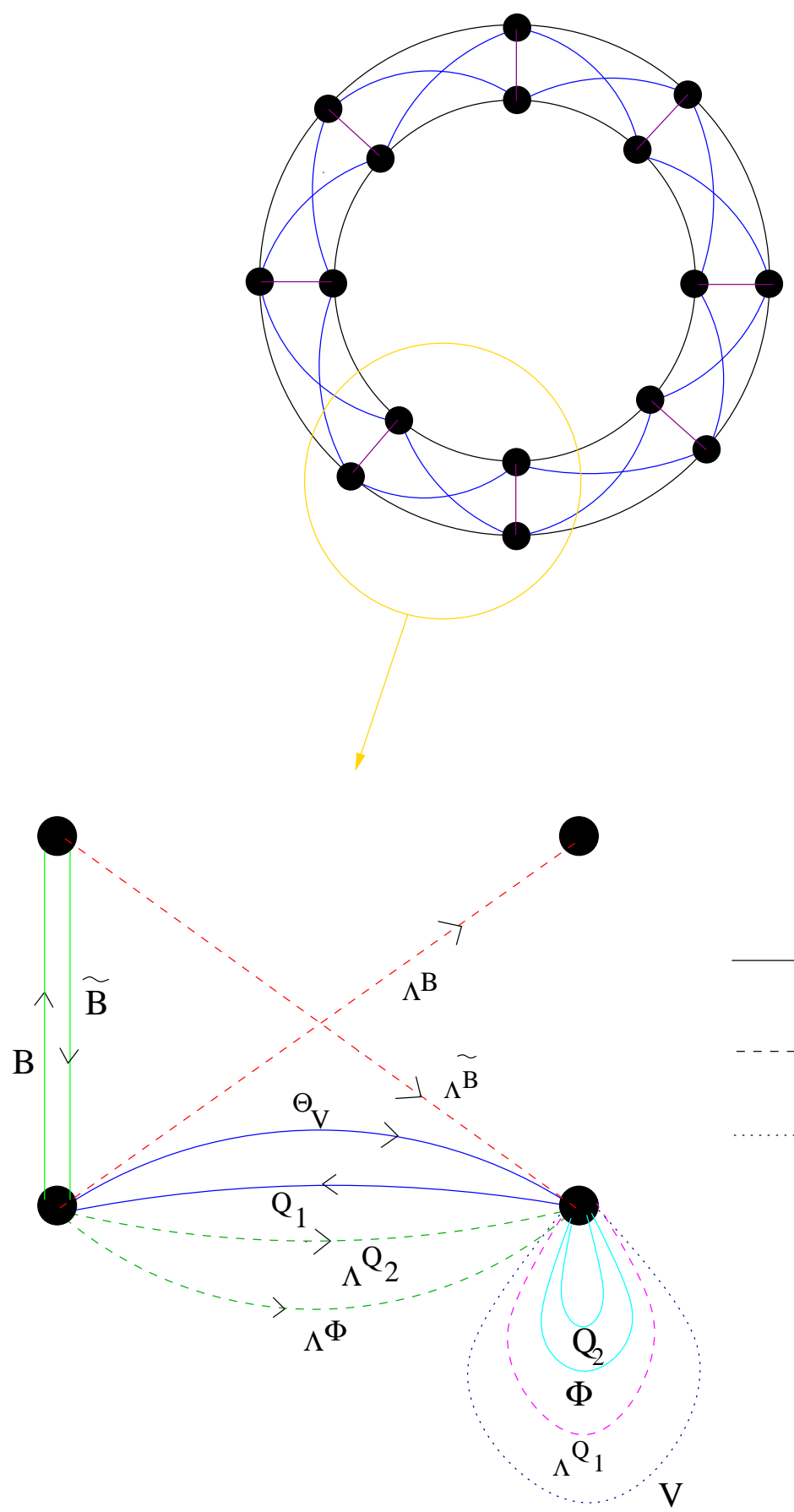

$(2,0)$ chiral multiplet

$(2,0)$ fermi multiplet

$(2,0)$ gauge multiplet

Figure 1: Quiver diagram for intersecting D3-branes at a $\mathbb{C}^{2} / \mathbb{Z}_{k}$ orbifold (with $k=8$ ). The nodes of the inner and outer circle are associated with the $S U\left(N^{\prime}\right)^{k}$ and $S U(N)^{k}$ gauge groups respectively. The parts which have not been drawn in the detailed "close-up" are easily inferred from the $\mathbb{Z}_{k}$ symmetry and by swapping D3 degrees of freedom with D3' degrees of freedom. 
In this expression the $\Lambda$ fields are $(2,0)$ Fermi multiplets, while the other fields are $(2,0)$ chiral multiplets. At the point in moduli space for which extra dimensions are generated, $\left\langle q_{j, j+1}^{1}\right\rangle=v I$ and $\left\langle s_{j, j+1}^{1}\right\rangle=v I$. Expanding about this point in the moduli space, the quadratic part of the superpotential is

$$
W_{\mathrm{D} 3-\mathrm{D} 3^{\prime}}=\left.g v \int d \theta^{+} \operatorname{tr}\left[\Lambda_{j, j+1}^{\tilde{B}}\left(B_{j}-B_{j+1}\right)+\left(\tilde{B}_{j+1}-\tilde{B}_{j}\right) \Lambda_{j, j+1}^{B}\right]\right|_{\bar{\theta}^{+}=0} .
$$

This superpotential can be viewed as a kinetic term in a compact lattice direction, corresponding to the circular quiver diagram, with $k$ sites and radius $R \sim \frac{k}{g v}$. Strictly speaking, there are additional contributions to the kinetic term arising from terms related to the superpotential by $(4,0)$ supersymmetry. These only change the radius by a numerical factor. By a discrete $\left(\mathbb{Z}_{k}\right)$ Fourier transform of modes localized at the intersection, one obtains Kaluza-Klein modes on the extra discrete circle. There is yet another discrete circle due to the S-duality of the D3-D3-orbifold system. Under S-duality $g \rightarrow k / g$, such that the radius of the other circle is $R_{D}=g / v$. The continuum limit which keeps both radii fixed is $k \rightarrow \infty$ with $g \sim \sqrt{k}$ and $v \sim \sqrt{k}$.

To determine whether the spoke degrees of freedom correspond to strings or particles in four dimensions, it is helpful to deform the theory by going to a point in the moduli space where they become massive. This is accomplished by setting $Y_{j, j+1}=(v+\Delta, v+\Delta)$ and $Y_{j, j+1}^{\prime}=(v-\Delta, v-\Delta)$, where $Y$ and $Y^{\prime}$ are the $S U(2)_{L}$ doublets of bifundamental scalars on the inner and outer rings of the quiver. Note that $q_{j, j+1}^{1}$ belongs to the doublet $Y_{j, j+1}$ while $s_{j, j+1}^{1}$ belongs to $Y_{j, j+1}^{\prime}$. The real parameter $\Delta$ must be scaled as $1 / \sqrt{k}$ to consistently generate two extra dimensions, but still has an effect as $k \rightarrow \infty$. The effective superpotential at this point in moduli space is

$$
\begin{aligned}
W & =\left.g v \int d \theta^{+} \operatorname{tr}\left[B_{j}\left(\Lambda_{-j, j+1}^{\tilde{B}}-\Lambda_{-j-1, j}^{\tilde{B}}\right)+\Lambda_{-j, j+1}^{B}\left(\tilde{B}_{j+1}-\tilde{B}_{j}\right)\right]\right|_{\bar{\theta}^{+}} \\
& +\left.g \Delta \int d \theta^{+} \operatorname{tr}\left[B_{j}\left(\Lambda_{-j, j+1}^{\tilde{B}}+\Lambda_{-j-1, j}^{\tilde{B}}\right)+\Lambda_{-j, j+1}^{B}\left(\tilde{B}_{j+1}+\tilde{B}_{j}\right)\right]\right|_{\bar{\theta}^{+}} .
\end{aligned}
$$

For large $k$ and fixed lattice momentum $n$, diagonalizing the mass matrix for the fundamental spoke degrees of freedom gives

$$
M^{2}=(g \Delta)^{2}+(n / R)^{2}
$$

where $n$ is the lattice momentum. For simplicity let us set $n=0$, so that $m=g \Delta$. The S-dual modes then have $m_{D}=\frac{k}{g} \Delta$. Since $m / m_{D}=R_{D} / R$, the fundamental spoke degrees of freedom should be interpreted as strings wrapping the cycle of radius $R_{D}$, while their

S-duals wrap the cycle of radius $R$. The string tension is $T=\frac{m}{2 \pi R_{D}}=\frac{m_{D}}{2 \pi R}=v \Delta$. As $\Delta \rightarrow 0$, we obtain a theory of tensionless strings.

\section{Matching R-symmetries and a 't Hooft Anomaly}

The M5-M5 intersection has $\mathcal{N}=2, d=4$ supersymmetry with $S U(2) \times U(1)$ R-symmetry. We shall now argue that the $S U(2)$ R-symmetry should be identified with the $S U(2)_{L}$ 
R-symmetry of the $(4,0)$ defect CFT. This is not a trivial identification, since the $S U(2)_{L}$ R-symmetry is apparently spontaneously broken at the point in moduli space required to generate two extra dimensions, whereas the $S U(2)$ R-symmetry of the M5-M5 intersections is only spontaneously broken when the M5-branes are transversely separated, corresponding to a non-zero string tension $T=\left|\vec{X}-\vec{X}^{\prime}\right|$. The quantity $\vec{X}-\vec{X}^{\prime}$ is a triplet under the $S U(2)$ R-symmetry. Note that the string tension in the $(4,0)$ description of M5-M5 intersection can be written in an $S U(2)_{L}$ invariant way as $T=\left|Y^{\dagger} \vec{\sigma} Y-Y^{\prime \dagger} \vec{\sigma} Y^{\prime}\right|$. This suggests the identification of $Y^{\dagger} \vec{\sigma} Y-Y^{\prime \dagger} \vec{\sigma} Y^{\prime}$ with $\vec{X}-\vec{X}^{\prime}$, which in turn requires the identification of $S U(2)_{L}$ with the $S U(2)$ R-symmetry of the M5-M5 system. This suggests that, for vanishing string tension, $S U(2)_{L}$ is unbroken as far as the non-trivial dynamics is concerned.

The only two-dimensional degrees of freedom of the $(4,0)$ defect CFT which are charged under $S U(2)_{L}$ are doublets of negative chirality. Consequently there is a 't Hooft anomaly in $S U(2)_{L}$. Note that such anomalies are protected quantities which are readily computable in the strong coupling limit. It is remarkable that the theory which (de)constructs the M5-M5 intersection is chiral, and it would be surprising if this chirality did not have consequences in the continuum limit. Assuming that the 't Hooft anomaly we have just discovered survives in this limit, it should be interpreted as an $S U(2)$ anomaly due to tensionless strings. While there are no local $S U(2)$ anomalies in four-dimensional quantum field theories, we know of nothing which excludes the possibility for four-dimensional theories of tensionless strings. Unfortunately, we can not yet state conclusively that the $S U(2)_{L}$ anomaly has a non-zero continuum limit.

Assuming that this anomaly exists in the continuum limit, it is somewhat similar in spirit to the known $\operatorname{Spin}(5)$ 't Hooft anomaly of the $(2,0)$ theory of parallel M5-branes 22, 23, 24, 25, 26, 27, 28]. The $\operatorname{Spin}(5)$ R-symmetry corresponds to the Lorentz symmetry of the five directions transverse the M5-branes. This symmetry is gauged by the coupling to gravity. It turns out there there is an anomaly in diffeomorphisms of the normal bundle due to the long wavelength Chern-Simons terms of eleven dimensional supergravity in the presence of magnetic (M5-brane) sources. Consistency requires that this anomaly is cancelled by a contribution due to the degrees of freedom propagating on the M5-brane. This gives an indirect derivation of the t' Hooft anomaly for the six-dimensional $(2,0)$ theory. At present there is no direct derivation in the non-abelian case. In principle, it should be possible to reproduce this anomaly from the (de)constructed description of the six-dimensional $(2,0)$ theory [18]. Unfortunately this is complicated by the fact that the Spin(5) R-symmetry is not manifest in this description, and is realized only in the continuum limit.

If there is an R-symmetry anomaly of the M5-M5 intersection due to tensionless strings, it should also be computable via the assumption of anomaly cancellation in Mtheory. The question of the existence of this anomaly is worth pursuing further, particularly in light of its relation to black hole entropy [24].

Acknowledgement The authors wish to thank G. Cardoso, A. Hanany, R. Helling, B. Ovrut, S. Ramgoolam, W. Skiba, D. Tong and J. Troost for helpful discussions. The research of J.E., Z.G. and I.K. is funded by the DFG (Deutsche Forschungsgemeinschaft) 
within the Emmy Noether programme, grant ER301/1-2. N.R.C. is supported by the DOE under grant DF-FC02-94ER40818, the NSF under grant PHY-0096515 and NSERC of Canada.

\section{References}

[1] S. Sethi, Nucl. Phys. B 523, 158 (1998) arXiv:hep-th/9710005.

[2] O. J. Ganor and S. Sethi, JHEP 9801 (1998) 007 arXiv:hep-th/9712071.

[3] A. Kapustin and S. Sethi, Adv. Theor. Math. Phys. 2, 571 (1998) arXiv:hepth/9804027].

[4] A. Karch and L. Randall, JHEP 0105, 008 (2001) arXiv:hep-th/0011156.

[5] A. Karch and L. Randall, JHEP 0106 (2001) 063 arXiv:hep-th/0105132.

[6] A. Karch and L. Randall, Phys. Rev. Lett. 87, 061601 (2001) arXiv:hep-th/0105108.

[7] O. DeWolfe, D.Z. Freedman and H. Ooguri, arXiv:hep-th/0111135.

[8] J. Erdmenger, Z. Guralnik and I. Kirsch, Phys. Rev. D 66, 025020 (2002) arXiv:hepth/0203020].

[9] P. Lee and J. w. Park, arXiv:hep-th/0203257.

[10] K. Skenderis and M. Taylor, JHEP 0206, 025 (2002) arXiv:hep-th/0204054.

[11] C. Bachas, J. de Boer, R. Dijkgraaf and H. Ooguri, JHEP 0206, 027 (2002) arXiv:hep-th/0111210.

[12] T. Quella and V. Schomerus, JHEP 0206, 028 (2002) arXiv:hep-th/0203161.

[13] D. Mateos, S. Ng and P. K. Townsend, JHEP 0207, 048 (2002) arXiv:hepth/0207136.

[14] N.R. Constable, J. Erdmenger, Z. Guralnik and I. Kirsch, arXiv:hep-th/0211222.

[15] A. Hanany and I. R. Klebanov, Nucl. Phys. B 482, 105 (1996) arXiv:hepth/9606136.

[16] S. Kachru, Y. Oz and Z. Yin, JHEP 9811, 004 (1998) arXiv:hep-th/9803050.

[17] N.R. Constable, J. Erdmenger, Z. Guralnik and I. Kirsch, arXiv:hep-th/0212136.

[18] N. Arkani-Hamed, A. G. Cohen, D. B. Kaplan, A. Karch and L. Motl, arXiv:hepth/0110146.

[19] C. Csaki, J. Erlich, J. Terning, arXiv:hep-th/0208095. 
[20] N. Arkani-Hamed, Th. Gregoire, J. Wacker, arXiv:hep-th/0101233.

[21] A. Hebecker, arXiv:hep-ph/0112230.

[22] E. Witten, J. Geom. Phys. 22, 103 (1997) arXiv:hep-th/9610234.

[23] D. Freed, J. A. Harvey, R. Minasian and G. W. Moore, Adv. Theor. Math. Phys. 2, 601 (1998) arXiv:hep-th/9803205.

[24] J. A. Harvey, R. Minasian and G. W. Moore, JHEP 9809, 004 (1998) arXiv:hepth/9808060].

[25] L. Bonora and M. Rinaldi, Nucl. Phys. B 578, 497 (2000) arXiv:hep-th/9912214.

[26] K. Lechner, P. A. Marchetti and M. Tonin, Phys. Lett. B 524, 199 (2002) arXiv:hepth/0107061].

[27] A. Boyarsky, J. A. Harvey and O. Ruchayskiy, Annals Phys. 301, 1 (2002) arXiv:hepth/0203154.

[28] K. A. Intriligator, Nucl. Phys. B 581, 257 (2000) arXiv:hep-th/0001205. 